\section{GUIDED VECTOR MODES OF EQUILATERAL THREE-CORE FIBRES}

Indexing terms: Optical fibres, Optical couplers, Fibre arrays, Polarisation

The six guided vector modes propagating on a equilateral three-core fibre with the component cores arrayed in an equilateral triangle have been calculated using a rigorous fullwave approach. It is shown that the polarisation patterns of these modes can also be obtained by manipulating the array modes determined using the coupled-mode theory.

Introduction: Three-core fibres or evanescent couplers made of three parallel single-mode fibres have recently received attention. ${ }^{1-3}$ In particular, the equilateral three-core fibres, whose component cores are arrayed in an equilateral triangle, have been analysed in References 2 and 3 based on symmetry arguments and the perturbation method, respectively. However, their results for the polarisation patterns and the mode splitting property of the vector modes are distinctly different. To realise the field properties of the vector modes propagating on this type of fibre, we have established a rigorous formulation* which is based on the expansion of the electromagnetic fields in terms of circular harmonics. In Reference 3 , the six polarisation patterns obtained using the perturbation analysis are shown to have a one-to-one similarity with the corresponding (fundamental and first-order) modal field patterns of a circularly symmetric fibre, respectively. The numerical results of our rigorous analysis have confirmed the validity of this perturbation analysis.

In this letter, rather than present the mathematical details of the full-wave method, we discuss, through the help of our mathematical analysis, how the vector mode patterns can be obtained from a linear combination of the coupled array modes and explain the mode splitting property on the basis of the correct polarisation patterns.
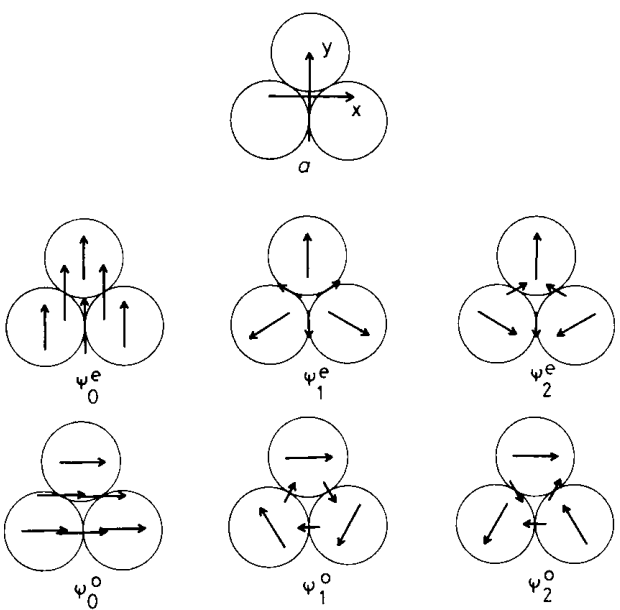

$b$

53971

Fig. 1

$a$ Cross-section and co-ordinate system of equilateral three-core fibre

$b$ Polarisation patterns for six independent vector modes

Field distributions and polarisation patterns: Consider the equilateral three-core fibres depicted in Fig. $1 a$. The component cores are identical, each single-mode, and arrayed in an equilateral triangle, and outside of them is an infinite uniform cladding. According to our mathematical analysis, the six independent vector mode polarisation patterns are as shown in Fig. 1b. There, we use six arrows to represent the orientation and relative strength of the electric fields at the three core centres and the three tangent points on the core/

- hUANG, H. S., and CHANG, H.-C.: 'Analysis of equilateral three-core fibres by circular harmonics expansion method', to be published cladding boundary. The upper three patterns of Fig. $1 b$ are the fundamental $\left(\psi_{0}^{e}\right)$ and first-order $\left(\psi_{1}^{e}\right.$ and $\left.\psi_{2}^{e}\right)$ even modes, and the lower three are the corresponding odd modes. Here, the term even (odd) denotes that the modes are symmetric (antisymmetric) with respect to the $y$-axis. Note that by rotating the modal fields schematically depicted in Fig. $1 b$ through an angle of $2 \pi / 3$ or $4 \pi / 3$, the polarisation or field pattern may retain invariant $\left(\psi_{1}^{e}\right.$ and $\psi_{1}^{o}$ ) or become variant (the other four modes) compared to the initial pattern in the $x-y$ co-ordinates.

In the following, we try to obtain the above results from a simpler consideration. According to the coupled-mode theory, ${ }^{4}$ the equilateral three-core fibre viewed as a coupler has amplitude distributions $(1,1,1)$ for the fundamental mode and $(2,-1,-1)$ and $(0,1,-1)^{2}$ for the degenerate first-order modes, respectively. Because the evanescent coupling takes place only between the modes having parallel or antiparallel polarisation, ${ }^{4}$ the coupled array modes are linearly polarised (LP) in any direction, as shown in Fig. 2.
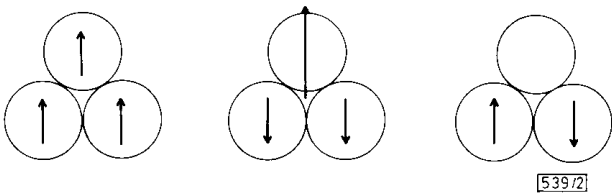

Fig. 2 Coupled array modes or linearly polarised $(L P)$ modes

The rotational symmetry of the equilateral three-core fibre demands that a mode which is invariant under a rotation of $2 \pi / 3$ or $4 \pi / 3$ exists. None of the LP modes shown in Fig. 2 satisfies this property. In fact, these four first-order LP modes are linear combinations of the first-order vector modes. The rotational symmetry also demands that the mode field can be decomposed into two parts: one is invariant under a rotation of $2 \pi / 3$, while the other is variant under the same rotation. Using rigorous analysis* it can be shown that by rotating the general solution of the wave equation for the even modes through an angle of $2 \pi / 3$ and $4 \pi / 3$, respectively, and summing these three field solutions, the rotationally variant part can be eliminated. Therefore, by summing the three field distributions, as shown in Fig. $3 a$, we obtain the second polarisation pattern depicted in Fig. $1 b$, which is rotation-invariant. The rotation-variant mode can be constructed in the manner as shown in Fig. $3 b$. The odd modes $\psi_{1}^{\circ}$ and $\psi_{2}^{\circ}$ can be obtained in a similar way. Finally, since the fundamental LP modes have a different propagation constant from that of the firstorder modes and each of them contains only the rotationally variant part, we conclude that the fundamental LP modes are themself the fundamental vector modes.

Vector mode splitting: Our analysis reveals that the two fundamental modes shown in Fig. $1 b$ are degenerate, and so are the two first-order modes $\psi_{2}^{e}$ and $\psi_{2}^{o}$. However, the modes $\psi_{1}^{e}$ and $\psi_{1}^{\circ}$ are nondegenerate to each other as well as to the modes $\psi_{2}^{e}$ and $\psi_{2}^{o}$. In Fig. 4 we plot the normalised propagation constant $\beta / n_{c l} k_{0}$ against $\delta\left(=1-n_{c l}^{2} / n_{c o}^{2}\right)$ curves for the structure depicted in the inset of the Figure to show the mode
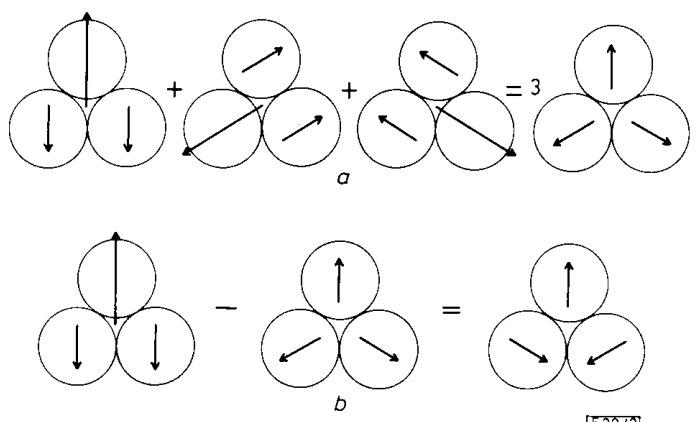

Fig. 3

a Schematic depiction of constructing mode $\psi_{1}^{e}$ from linear combination of LP modes

$b$ Schematic depiction of constructing mode $\psi_{2}^{e}$ from linear combination of LP mode and mode $\psi_{1}^{e}$ 
splitting behaviour, where $n_{c l}$ and $n_{c o}$ are the refractive indices of the cladding and core, respectively. It is noted that when the equilateral three-core fibres are of weak guidance $(\delta \simeq 0)$, the splitting of the four first-order modes is insignificant and thus our manipulation of these mode patterns in the last section is applicable. However, as the guidance becomes stronger, the rigorous full-wave analysis should be employed.

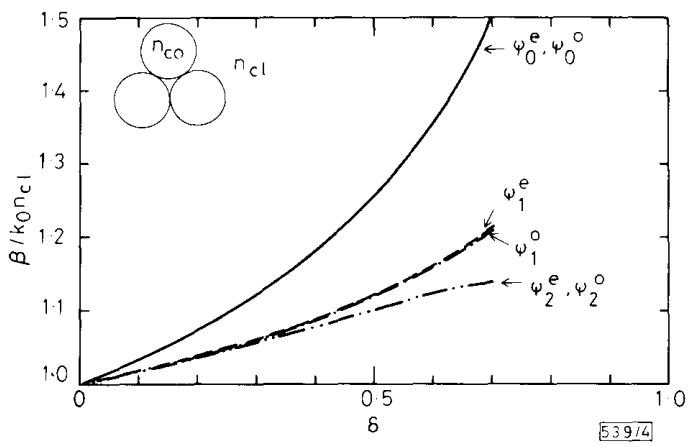

Fig. 4 Plot of normalised propagation constant $\beta / k_{0} n_{c l}$ as function of $\delta$ where $\delta=1-n_{c l}^{2} / n_{c o}^{2}$

$V=2, k_{0}=4.833 \mu \mathrm{m}^{-1}, n_{c l}=1.455$

The nondegeneracy of the modes $\psi_{1}^{e}$ and $\psi_{1}^{o}$ can be viewed as resulting from their invariance when rotated through an angle of $2 \pi / 3$ or $4 \pi / 3$. In contrast, a new mode obtained by rotating $\psi_{2}^{e}$ clockwise through an angle of $2 \pi / 3$ can be shown to be simply a linear combination of the form $-(1 / 2) \psi_{2}^{e}+(\sqrt{ }(3) / 2) \psi_{2}^{o}$. Thus, the two modes $\psi_{2}^{e}$ and $\psi_{2}^{o}$ should have the same propagation constant. The same argu- ment can also be applied to the fundamental modes, and so they have the same propagation constant.

The last thing to be noted is that since the modes $\psi_{0}^{e}$ and $\psi_{0}^{o}$ are degenerate, any linear combination of them can be a mode. Thus, the fundamental mode can be polarised in any direction, as stated in Reference 3. Also, any linear combination of the modes $\psi_{2}^{e}$ and $\psi_{2}^{o}$ can be a mode.

Conclusions: We have presented the mode properties of the equilateral three-core fibres obtained using a rigorous fullwave method, and have confirmed the results of Reference 3 based on an approximate analysis. We have also shown that the polarisation patterns of the vector modes can be deduced from the array modes provided by the coupled-mode theory.

Acknowledgment: This work was supported by the National Science Council of the Republic of China under grants NSC77-0608-E002-01 and NSC77-0608-E002-04.

H. S. HUANG

29th September 1988

H.-C. CHANG

Department of Electrical Engineering

National Taiwan University

Taipei, Taiwan, Republic of China

\section{References}

1 SHEEM, $\mathbf{s .}$ K.: 'Optical fiber interferometers with [ $3 \times 3]$ directional couplers: analysis', J. Appl. Phys., 1981, 52, pp. 3865-3872

2 BLACK, R. J., GAGNON, L., YOUNGQUIST, R. C., and WENTWORTH, R. H.: 'Modes of evanescent $3 \times 3$ couplers and three-core fibres', Electron. Lett., 1986, 22, pp. 1311-1313

3 STEVENSON, A. J., and LOVE, J. D.: 'Vector modes of six-port couplers', ibid., 1987, 23, pp. 1011-1013

4 SNYDER, A. W.: 'Coupled-mode theory for optical fibers', J. Opt. Soc. Am., 1972, 62, pp. 1267-1277

\section{APPLICATION OF ON-SURFACE RADIATION CONDITION TO ELECTROMAGNETIC SCATTERING BY CONDUCTING STRIP}

\author{
Indexing terms: EM waves, Scattering
}

The scattering of a plane electromagnetic wave by an infinite perfectly conducting strip is studied using the on-surface radiation condition technique. For transverse magnetic (TM) incidence the presence of new contributions to the edge diffracted fields is demonstrated. It is shown that inclusion of these contributions greatly improves the accuracy of the scattered field predictions.

Introduction: A new formulation of electromagnetic scattering has been reported by Kriegsmann, Taflove and Umashankar. ${ }^{1}$ Central to this scheme is the use of an approximate boundary condition obtained by imposing a form of the radiation condition directly on the surface of the scatterer. This so-called 'on-surface radiation condition' (OSRC) approach is less computationally intensive than an integral equation or other appropriate method and promises to be a valuable tool in the resonant frequency region.

In this letter, the application of the OSRC technique to scattering by an infinite conducting strip is re-examined. The results of Reference 1 are shown to be incomplete for the case of the scattering of an E-polarised (TM) plane wave. Extra contributions to the scattered field are identified and may be viewed as being due to diffraction at the edges. Inclusion of the new edge waves is shown to improve the accuracy of the OSRC solution.

In the following Section, we analyse the problem of the scattering of a TM plane wave incident normally upon a conducting strip by viewing the scatterer as an elliptic cylinder with vanishing minor axis.

Analysis: Consider the electromagnetic scattering of a plane wave of unit amplitude incident normally upon an infinite perfectly conducting elliptic cylinder (see Fig. 1). The major and minor axes of the ellipse are of lengths $2 a$ and $2 b$, respec- tively. It is convenient to use elliptic cylinder co-ordinates $(u, v, z)$ and circular cylinder co-ordinates $(r, \phi, z)$ where

$$
\begin{aligned}
& x=l \cosh u \cos v=r \cos \phi \\
& y=l \sinh u \sin v=r \sin \phi
\end{aligned}
$$

with $0 \leq u<\infty, 0 \leq v<2 \pi$ and $l^{2}=a^{2}-b^{2}$. The surface of the scatterer is given by $u=u_{0}$, with $a=l \cosh u_{0}$ and $b=$ $l \sinh u_{0}$. In the limit $u_{0} \rightarrow 0(a \rightarrow l, b \rightarrow 0)$ the elliptic cylinder degenerates to the strip $-l \leq x \leq l, y=0$.

The incident plane wave approaches from the $\phi=\pi-\alpha$ direction and is assumed to be time-harmonic. All fields will then exhibit the same time dependence, chosen to be $\exp (-j \omega t)$, which will be suppressed throughout. For an Epolarised incident plane wave the total electric field at a point external to the cylinder with position vector $r$ is given by

$$
E(\boldsymbol{r})=(0,0, E(\boldsymbol{r}))=\left(0,0, E^{i}(\boldsymbol{r})+E^{\mathrm{s}}(\boldsymbol{r})\right)
$$

where $E^{i}(\boldsymbol{r})$ and $E^{s}(\boldsymbol{r})$ denote the incident and scattered fields, respectively. Since the scatterer is perfectly conducting the condition

$E^{i}\left(\boldsymbol{r}^{\prime}\right)+E^{s}\left(\boldsymbol{r}^{\prime}\right)=0$

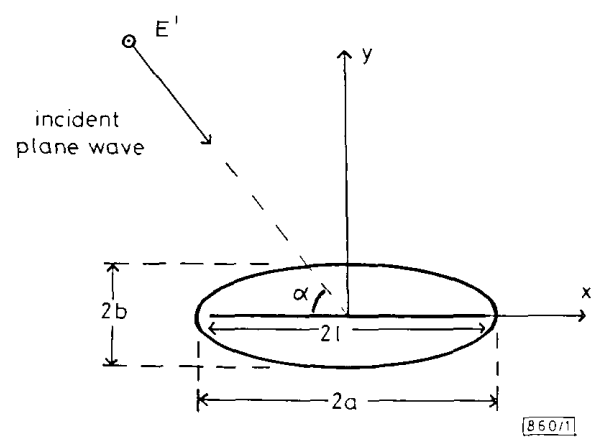

Fig. 1 Plane wave incident on conducting elliptic cylinder 